\title{
19. Observations of Polarization of Solar Radio Bursts
}

\author{
By Takeo Hatanaka, Shigemasa SuzUKI, and Atsushi TsuchiYA \\ Tokyo Astronomical Observatory, University of Tokyo \\ (Comm. by Y. Hagihara, M.J.A., Feb. 18, 1955)
}

1. Introduction. The polarization of solar radio bursts of Type I in meter wavelength region has been thought to be circular without any detailed investigations. ${ }^{1)}$ While working at the School of Electrical Engineering, Cornell University, during 1952 and 1953, one of the present authors (T. H.) has analyzed the tapes taken by the Cornell radio-astronomy groups both at Ithaca, N.Y., and at Sacramento Peak, N. M., at the frequency of $200 \mathrm{Mc} / \mathrm{s}$ and reached the conclusion that the polarization of bursts which are associated with active sunspots (Type I bursts) is elliptic in general whose shape and orientation depend on the position of the source on the solar disk. ${ }^{23}$ The number of the observed components available then, however, was three, i. e. the linearly polarized component in horizontal direction in the celestial sphere observed at Ithaca, and two circularly polarized components of opposite sense of rotation observed at Sacramento Peak. Since the number of independent components necessary to determine the complete state of polarization is four, the conclusion reached then was not conclusive. Moreover, the circularly polarized components were observed in such a way that right-handed and left-handed circularly polarized observations were switched from one to the other in every ten minutes. Therefore the foregoing analysis had to be done not for each burst but on a statistical basis.

The necessity for a new radio polarimeter was discussed with the staffs at Cornell. It seemed that the polarimeter is to observe at least four independent components of polarization of bursts. It was also suggested that such a polarimeter is to be designed on a time-sharing scheme in order to avoid inevitable errors which were encountered during the course of the foregoing analysis because of the difficulties in calibrating different receiving systems.

2. Time-Sharing Radio Polarimeter. A new radio polarimeter on a time-sharing scheme has been designed and constructed at the Tokyo Astronomical Observatory for use at $200 \mathrm{Mc} / \mathrm{s}$. A pair of crossed dipoles are put at the focus of the 10 meter dish of equatorial mounting recently built in the Observatory. The inclinations of the two dipoles are $\pm 45^{\circ}$ respectively from the north in the celestial sphere. Suitable phase retardations, as shown in Figs. 1 and 2, are put in the circuits at the intermediate frequency stage,

1) Cf. "The Sun" Ed. by G. P. Kuiper, Chapter 7, "Solar Radio Emission" by J. L. Pawsey and S. F. Smerd, 1954.

2) T. Hatanaka: Research Report EE No. 179, Cornell University, 1953. 


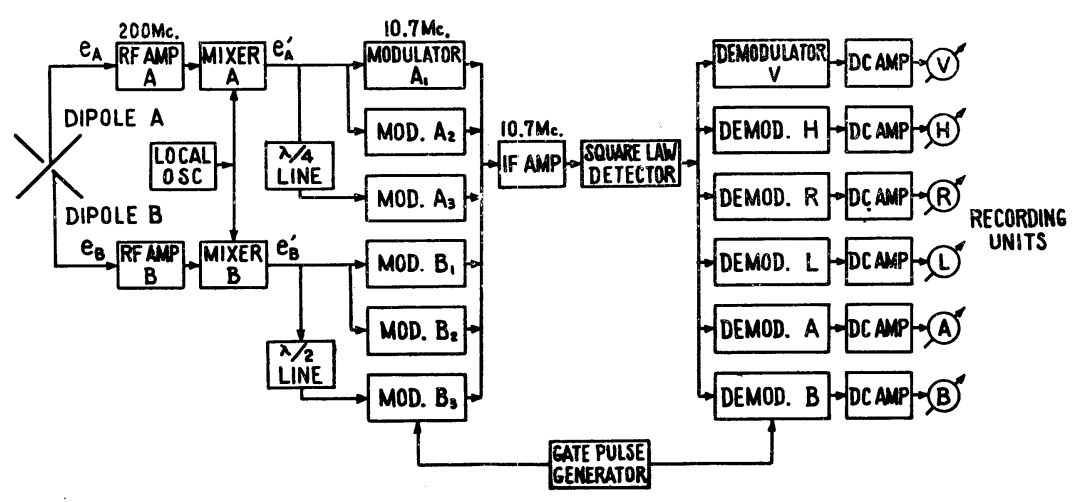

Fig. 1. Block diagram of the circuit

and combined so as to give two linearly polarized components, i.e. one in the northsouth (or vertical) direction and the other in the east-west (or horizontal) direction, and two circularly polarized components in right-handed and left-handed senses of rotation. (Let us call these four as $v, h, r$, and $l$ respectively.) These four components together with two linearly polarized components in the directions of the original dipoles (which we call $a$ and $b$ ) are switched in cyclic manner with the duration of $1 / 1600 \mathrm{sec}$. for each. An electronic switching system with eight gates by using

6 AS6 pentodes is employed so that the duration of one cycle is $1 / 200$ sec. with two gates not being used at present. The duration of one cycle seems to be sufficiently short compared with the duration of a burst, which is of the order of a few or several seconds, and practically a set of simultaneous observations of six components of 
polarization of a burst is achieved with a common receiving system.

By a demodulator, synchronously operated by the electronic switch, the separate informations of each component are gathered together and fed to a six channel pen-recorder. The time constant of the total system is set to 0.5 sec. for normal observation and changed to $2.5 \mathrm{sec}$. for the calibration or for the measurement of the flux of the sun. The overall pre-detector bandwidth is approximately $100 \mathrm{kc} / \mathrm{s}$. The tapes used in the present investigation were run at a speed of $60 \mathrm{~mm} / \mathrm{min}$, but are slowed down to $4 \mathrm{~mm} / \mathrm{min}$ during the quiet period.

The calibrations have been put at every one hour, or at least at every three hours, by means of built-in noise generators.

3. Observation and Reduction. The polarimeter has been put to field tests since October, 1954, and finally used for the solar observations since early in December. Our first record of bursts have been taken on December 16, 1954.
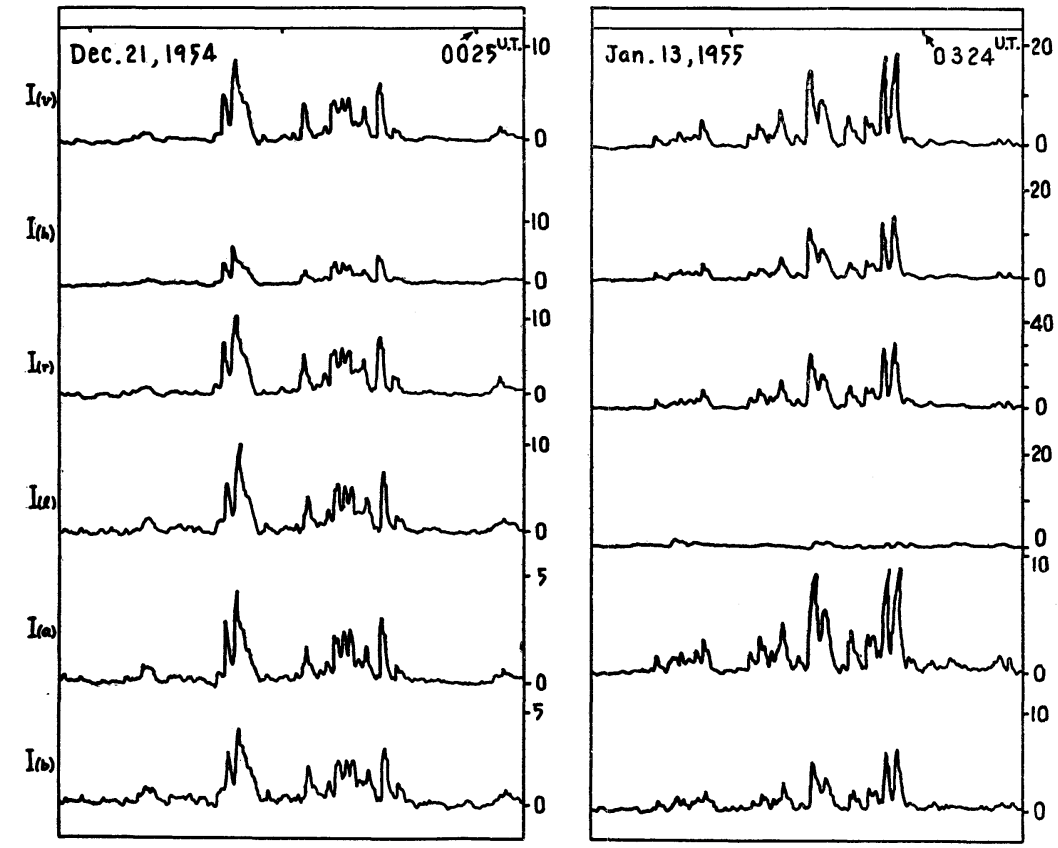

Fig. 3. Examples of the records of bursts (1/3 of the original size)

Fig. 3 shows some examples of the records of bursts used in the present analysis. From top to bottom they are the records of the components of $v, h, r, l, a$, and $b$. The time marks are put at the extreme top at every one minute, and the time goes from left to right on the tape. Normal observation hours cover from $0^{\text {h }}$ U.T. to $6^{\mathrm{h}}$ U.T.

Our method of analysis is to deduce Stokes' parameters") directly

3) Cf. S. Chandrasekhar: "Radiative Transfer", 1951. 
from the observation. If we write the power, which is calibrated by means of the noise generators, in each component by $I(v), I(h)$, etc. it is easily shown that the Stokes' parameters, $I, Q, U$, and $V$ are given by

$$
\begin{aligned}
I=\frac{1}{2}\{I(v)+I(h)\} & =\frac{1}{2}\{I(r)+I(l)\}=I(a)+I(b) \\
Q & =I(a)-I(b) \\
U & =\frac{1}{2}\{I(v)-I(h)\} \\
V & =\frac{1}{2}\{I(r)-I(l)\}
\end{aligned}
$$

when the reference co-ordinates are taken in the direction of $a$ and $b$. The first of the eqs. (1) serves for the check.

When most of the bursts during some time intervals have almost identical polarization state, the solution is obtained for their mean values, otherwise the parameters are solved for each burst or by gathering bursts into several groups.

It is known that when the radiation is completely polarized, i.e. elliptically polarized,

$$
I=\left(Q^{2}+U^{2}+V^{2}\right)^{\frac{1}{2}}
$$

It is found that the condition (2) is not always satisfied for the bursts observed, and the right-hand side of eq. (2) sometimes becomes much smaller than the left-hand side (December 21, 1954, in Table I, for example). This means that such radiation is to be considered to be the mixture of a randomly polarized component with the Stokes' parameters $\left(I_{r}, 0,0,0\right)$ and a elliptically polarized component with $\left(I_{e}, Q, U, V\right)$, where

$$
\begin{gathered}
I_{e}=\left(Q^{2}+U^{2}+V^{2}\right)^{\frac{1}{2}}, \\
I_{r}=I-I_{e} .
\end{gathered}
$$

The ratio $I_{e} / I$ gives the degree of polarization. The ratio, $p$, of the minor axis to the major axis and the inclination of the major axis measured from the vertical direction $\left(\chi^{\prime}\right)$ of the elliptic component can be determined by the standard method. $\chi^{\prime}$ is measured eastwards from the north when projected on the celestial sphere.

Table I. Polarization of solar radio bursts at $200 \mathrm{Mc} / \mathrm{s}$

(Dec. 16-22, 1954)

\begin{tabular}{c|c|cccc}
\hline Date & Hours & \multicolumn{4}{c}{ Polarization } \\
Dec. 1954 & (U. T.) & $I_{e} / I$ & $I_{r} / I$ & $\chi^{\prime}$ & $p$ \\
\hline 16 & $0^{\mathrm{h}}-1^{\mathrm{h}}$ & 0.82 & 0.18 & $52^{\circ}$ & $0.75 \mathrm{R}$ \\
& & 0.48 & 0.52 & 60 & $0.72 \mathrm{R}$ \\
& $2-3$ & 0.78 & 0.22 & 59 & $0.71 \mathrm{R}$ \\
& & 0.46 & 0.54 & 24 & $0.50 \mathrm{R}$ \\
& $3-4$ & 0.86 & 0.14 & 59 & $0.70 \mathrm{R}$ \\
17 & $4-5$ & 0.86 & 0.14 & 62 & $0.70 \mathrm{R}$ \\
18 & $5-6$ & 0.91 & 0.09 & 58 & $0.66 \mathrm{R}$ \\
19 & $0-1$ & 0.75 & 0.25 & 74 & $0.70 \mathrm{R}$ \\
20 & $0-2$ & 0.94 & 0.06 & 35 & $0.70 \mathrm{R}$ \\
21 & $3-4$ & 0.62 & 0.38 & 83 & $0.60 \mathrm{R}$ \\
22 & $4-5$ & 0.52 & 0.48 & 58 & $0.75 \mathrm{R}$ \\
& $0-1$ & 0.11 & 0.89 & 20 & $0.35 \mathrm{R}$ \\
& $5-6$ & 0.61 & 0.39 & 77 & $0.08 \mathrm{R}$ \\
\hline
\end{tabular}


Table II. Polarization of solar radio bursts at $200 \mathrm{Mc} / \mathrm{s}$

(Jan. 9-17, 1955)

\begin{tabular}{|c|c|c|c|c|c|}
\hline \multirow{2}{*}{$\begin{array}{c}\text { Date } \\
\text { Jan. } 1955\end{array}$} & \multirow{2}{*}{$\begin{array}{l}\text { Hours } \\
\text { (U. T.) }\end{array}$} & \multicolumn{4}{|c|}{ Polarization } \\
\hline & & $I_{e} / I$ & $I_{r} / I$ & $\chi^{\prime}$ & $p$ \\
\hline 9 & $\begin{array}{c}0^{\mathrm{h}}-1^{\mathrm{h}} \\
1-2\end{array}$ & $\begin{array}{r}\sim_{0.95}^{1.0} \\
0.95\end{array}$ & $\widetilde{\sim 0}$ & $\begin{array}{l}45^{\circ} \\
40\end{array}$ & $\begin{array}{l}0.70 \mathrm{R} \\
0.70 \mathrm{R}\end{array}$ \\
\hline 10 & $\begin{array}{l}2-3 \\
3-4 \\
4-5 \\
5-6\end{array}$ & $\begin{array}{l}0.90 \\
0.95 \\
0.93 \\
0.95\end{array}$ & $\begin{array}{l}0.10 \\
0.05 \\
0.07 \\
0.05\end{array}$ & $\begin{array}{l}45 \\
45 \\
32 \\
32\end{array}$ & $\begin{array}{l}0.70 \mathrm{R} \\
0.70 \mathrm{R} \\
0.69 \mathrm{R} \\
0.71 \mathrm{R}\end{array}$ \\
\hline 11 & $\begin{array}{l}0-1 \\
3-4\end{array}$ & $\begin{array}{l}0.96 \\
0.90\end{array}$ & $\begin{array}{l}0.04 \\
0.10\end{array}$ & $\begin{array}{l}20 \\
20\end{array}$ & $\begin{array}{l}0.77 \mathrm{R} \\
0.75 \mathrm{R}\end{array}$ \\
\hline 12 & $\begin{array}{l}0-1 \\
1-2 \\
2-3 \\
4-5 \\
5-6\end{array}$ & $\begin{array}{l}0.95 \\
0.85 \\
0.90 \\
0.91 \\
0.89\end{array}$ & $\begin{array}{l}0.05 \\
0.15 \\
0.10 \\
0.09 \\
0.11\end{array}$ & $\begin{array}{l}70 \\
75 \\
70 \\
74 \\
60\end{array}$ & $\begin{array}{l}0.73 \mathrm{R} \\
0.78 \mathrm{R} \\
0.74 \mathrm{R} \\
0.70 \mathrm{R} \\
0.83 \mathrm{R}\end{array}$ \\
\hline 13 & $0-1$ & $0.50-0.60$ & $0.50-0.40$ & $70-85$ & $0.32-0.55 \mathrm{R}$ \\
\hline 14 & $1-2$ & 0.95 & 0.05 & 63 & $0.73 \mathrm{R}$ \\
\hline 15 & $5-6$ & $\sim 1.0$ & 0 & 80 & $0.60-0.70 \mathrm{R}$ \\
\hline 16 & $\begin{array}{l}0-1 \\
2-3\end{array}$ & $\begin{array}{l}0.85-0.95 \\
0.85-0.95\end{array}$ & $\begin{array}{l}0.15-0.05 \\
0.15-0.05\end{array}$ & $\begin{array}{l}50-60 \\
55-75\end{array}$ & $\begin{array}{l}0.65-0.80 R \\
0.65-0.80 R\end{array}$ \\
\hline 17 & $0-1$ & $0.42-0.52$ & $0.58-0.48$ & $55-60$ & $0.50-0.80 \mathrm{R}$ \\
\hline
\end{tabular}

Tables I and II give the results analyzed so far for the active periods in December, 1954, and January, 1955, respectively. When the bursts are analyzed by dividing into several groups, the solution for each group is given in the order of activity in each time interval. No observation has been carried out from December 24 to January 6 owing to minor readjustments of the receiver.

The states of polarization are illustrated schematically in Fig. 4 . These diagrams are drawn in normalized form in such a way that the area of each component, randomly or elliptically polarized, represents the relative intensity $I_{r} / I$ or $I_{e} / I$, and the sum of the two is the same for each day.

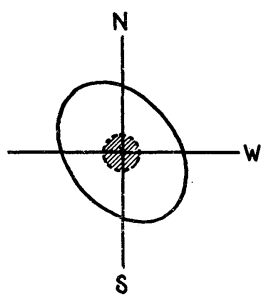

Dec. $18,00^{h}-02^{h}$

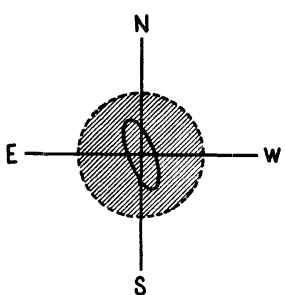

Dec. $21,00^{h}-01^{h}$ (1954)

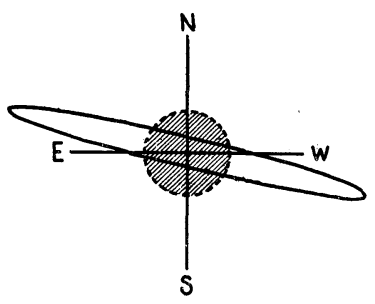

Dec. 22, 0530

Fig. 4. Schematic illustration of the states of polarization

4. Discussions. The foregoing result shows that the state of polarization is not always the same, but varies from time to time. It would be of interest, especially for the study on the mechanism of emission, to investigate the change in relation to the sunspot observation. Table III gives the data of active sunspots during these 
Table III. List of active sunspots from December 15, 1954, to January 20, 1955

\begin{tabular}{rrrrr|r|c}
\hline $\begin{array}{c}\text { Group } \\
\text { No. }\end{array}$ & $\begin{array}{c}\text { First } \\
\text { seen }\end{array}$ & $\begin{array}{c}\text { Central } \\
\text { meridian } \\
\text { passage }\end{array}$ & $\begin{array}{c}\text { Last } \\
\text { seen }\end{array}$ & $\begin{array}{c}\text { Mean } \\
\text { latitude }\end{array}$ & $\begin{array}{c}\text { Type at } \\
\text { maximum }\end{array}$ \\
\hline $1954-42$ & Dec. 15 & Dec. 15 & Dec. 21 & +33.5 & C (D) \\
44 & 29 & 30 & Jan. 5 & -24 & D \\
$1955-2$ & Jan. 4 & Jan. 8 & 14 & +20 & E \\
5 & & 13 & 20 & +36 & H \\
\hline
\end{tabular}

periods kindly prepared by Dr. M. Notuki of the Observatory. By putting off more detailed discussions to future, the following points. are remarked here.

(a) The degree of polarization $\left(I_{e} / I\right)$ is greater than $85 \%$ in most cases but is about $10 \%$ in some occassions, such as on December 21 and January 19. It seems that $I_{e} / I$ decreases after several days of high value of $I_{e} / I$. The ellipticity $(p)$ is not constant, but, when compared with the sunspot data, it is pointed out that the decrease in $p$ is observed when the associated sunspot approaches to the limb of the sun (December 21 and 22, for example). The value of $\chi^{\prime}$ varies in a way much more erratic than $I_{e} / I$ and $p$.

(b) Although there exists no satisfactory theory on the emission mechanism of bursts at present, it is generally accepted that the magnetic field above a sunspot plays an important role in the emission and/or in the propagation of Type I bursts. It is tempting to. assume that the radio waves emitted in the presence of strong magnetic field are circularly polarized when viewed along the direction of the magnetic field. If so, the variation of the ellipticity discussed above is in accordance with this simple picture if the direction of the magnetic field is normal to the sun's surface in a layer high above a sunspot. The degree of polarization, then, can be understood to represent the degree of regularity of the magnetic field. It seems natural to suppose that such regularity would be destroyed when the turbulence in the solar atmosphere becomes effective. The irregular variation of $\chi^{\prime}$ seems to be due to the rotation of the axis of the ellipse by the Faraday effect in the earth's ionosphere and possibly in the solar atmosphere.

5. Summary. A new radio polarimeter is described, which is designed and constructed at the Tokyo Astronomical Observatory to observe the extra-terrestrial radio emission at $200 \mathrm{Mc} / \mathrm{s}$. It consists of a pair of crossed dipoles attached to the 10 meter paraboloid with suitable phase-retardations and an electronic switch so as to give the informations of six components of polarization simultaneously.

The results of the analysis of solar radio bursts in December, 1954, and January, 1955, are presented. It is pointed out that the radiation of a burst is, in general, a mixture of two components, one randomly and the other elliptically polarized. The degree of polarization, or the fraction of the elliptically polarized component, 
seems to decrease in several days, while the ellipticity of the polarization ellipse depends upon the position of the source on the solar disk and the inclination of the major axis is subject to the Faraday effect in the earth's ionosphere.

Details of the observing system and results of further analysis will be published in the Publications of the Astronomical Society of Japan.

The authors wish to express their hearty thanks to Prof. Y. Hagihara, Director, for his encouragement and interest. Their thanks are due to the staffs of the Observatory for their discussions and kind collaboration. One of the authors (T.H.) thanks to Drs. C. R. Burrows and W. E. Gordon, Cornell University, for their kind discussions. The construction and observation were assisted by Mr. N. Shibuya and the reduction by Miss. H. Kurai and Mr. S. Takeda. 